\title{
Research ethics and refugee health: a review of reported considerations and applications in published refugee health literature, 2015-2018
}

Emma E. Seagle 1,2,3, Amanda J. Dam, ${ }^{1,4}$, Priti P. Shah ${ }^{1,3}$, Jessica L. Webster ${ }^{1,3}$, Drue H. Barrett ${ }^{5}$, Leonard W. Ortmann ${ }^{5}$, Nicole J. Cohen ${ }^{1}$ and Nina N. Marano ${ }^{1 *}$ (iD

\begin{abstract}
Introduction: Public health investigations, including research, in refugee populations are necessary to inform evidence-based interventions and care. The unique challenges refugees face (displacement, limited political protections, economic hardship) can make them especially vulnerable to harm, burden, or undue influence. Acute survival needs, fear of stigma or persecution, and history of trauma may present challenges to ensuring meaningful informed consent and establishing trust. We examined the recently published literature to understand the application of ethics principles in investigations involving refugees.

Methods: We conducted a preliminary review of refugee health literature (research and non-research data collections) published from 2015 through 2018 available in PubMed. Article inclusion criteria were: participants were refugees, topic was health-related, and methods used primary data collection. Information regarding type of investigation, methods, and reported ethics considerations was abstracted.

Results: We examined 288 articles. Results indicated 33\% of investigations were conducted before resettlement, during the displacement period (68\% of these were in refugee camps). Common topics included mental health $(48 \%)$ and healthcare access (8\%). The majority (87\%) of investigations obtained consent. Incentives were provided less frequently (23\%). Most authors discussed the ways in which community stakeholders were engaged (91\%), yet few noted whether refugee representatives had an opportunity to review investigational protocols (8\%). Cultural considerations were generally limited to gender and religious norms, and 13\% mentioned providing some form of post-investigation support.

\footnotetext{
* Correspondence: nbm8@cdc.gov

*The findings and conclusions in this report are those of the author(s) and do not necessarily represent the official position of the Centers for Disease Control and Prevention.

'Division of Global Migration and Quarantine, Centers for Disease Control and Prevention, 1600 Clifton Road, MS EO3, Atlanta, GA 30333, USA

Full list of author information is available at the end of the article
}

(c) The Author(s). 2020, corrected publication 2022. Open Access This article is licensed under a Creative Commons Attribution 4.0 International License, which permits use, sharing, adaptation, distribution and reproduction in any medium or format, as long as you give appropriate credit to the original author(s) and the source, provide a link to the Creative Commons licence, and indicate if changes were made. The images or other third party material in this article are included in the article's Creative Commons licence, unless indicated otherwise in a credit line to the material. If material is not included in the article's Creative Commons licence and your intended use is not permitted by statutory regulation or exceeds the permitted use, you will need to obtain permission directly from the copyright holder. To view a copy of this licence, visit http://creativecommons.org/ licenses/by/4.0/. The Creative Commons Public Domain Dedication waiver (http//creativecommons.org/publicdomain/zero/1. 0/) applies to the data made available in this article, unless otherwise stated in a credit line to the data. 


\begin{abstract}
(Continued from previous page)
Conclusions: Our analysis is a preliminary assessment of the application of ethics principles reported within the recently published refugee health literature. From this analysis, we have proposed a list of best practices, which include stakeholder engagement, respect for cultural norms, and post-study support. Investigations conducted among refugees require additional diligence to ensure respect for and welfare of the participants. Development of a refugee-specific ethics framework with ethics and refugee health experts that addresses the need for stakeholder involvement, appropriate incentive use, protocol review, and considerations of cultural practices may help guide future investigations in this population.
\end{abstract}

Keywords: Ethics, Research, Framework, Refugee, Health

\section{Introduction}

The UN Refugee Agency (UNHCR) estimates that, in 2018, there were over 25 million refugees worldwide, with nearly 37,000 people daily forced by conflict or persecution to flee their homes [1]. Complex migration patterns and poor access to healthcare often result in physical and mental health concerns throughout all stages of the refugee experience, including flight, asylum, and resettlement or return to their home country. Furthermore, refugees often have disparate risks of disease. These factors may contribute to health disparities among refugee populations and between refugees and nonrefugees, which underscore the importance of conducting research specific to refugees and ensuring their representation in analyses (i.e., ensuring refugees are not excluded from analyses solely due to their vulnerability) [2]. High-quality data from such assessments are needed to develop evidence-based interventions and services and to guide national and international health policies.

The unique challenges refugees face can make them especially vulnerable to harm, burden, or undue influence in investigational settings [3-7]. For instance, refugees' vulnerability is heightened by stress of relocation, family separation, exposure to violence or torture, uncertainty about the future, and prior or ongoing trauma [8]. Refugees also often lack the same rights as citizens in their countries of asylum, potentially resulting in limited political protections from maltreatment and limited access to healthcare, employment, or education [4]. Loss of assets during flight can also contribute to economic hardship and increase dependence on free social services for asylum-seekers. Some refugees are subject to continued oppression, restrictions on their liberty of movement, and gross power imbalances within refugee camps [6]. Additionally, low literacy (including health literacy) levels in some refugees can result in communication barriers and low understanding or misinterpretation of the investigation process $[6,9]$.

Ultimately, tension exists between the need for data to inform evidence-based practices and the need to protect vulnerable refugee populations from research-related risks [4]. The inherent dangers and extreme circumstances affecting people experiencing conflict and forced migration can make it difficult to simultaneously collect quality data and protect the rights of individuals with adherence to the highest ethical standards [7]. However, to exclude refugees from research or public health investigations because of their vulnerability violates the codes of justice and fairness [10], because evidence obtained from such investigations could inform targeted interventions, validate models of health service delivery, and ultimately protect the well-being of these individuals $[3,4,7,11]$. Additionally, to only conduct research in non-refugee populations, even those with similar demographic characteristics, could provide an inaccurate and inadequate representation $[3,12,13]$. While there is clear justification for the need to conduct such investigations, it remains critical for investigators-as well as reviewers responsible for approving investigations-to address the myriad of complex ethical challenges present before, during, and after investigations [11].

Complex challenges are present throughout the refugee's journey and may vary depending on whether the refugee is displaced (either internally or in a country of asylum) or has been formally resettled in a country that has granted permanent settlement. These challenges introduce complexities to conducting ethical investigations, including ensuring meaningful and voluntary consent, preventing real or perceived coercion to participate, minimizing undue influence (including economic), mitigating burden, and accounting for power imbalances between the investigators and participants $[3,5,7]$. For instance, participants could be motivated by the potential for economic gains or other tangible benefits (e.g., access to resources that are otherwise scarce), fear of consequences of not participating (e.g., when investigators in conflict areas are accompanied by armed guards, or fear of potential loss of benefits), or the possible misunderstanding that participation could help accelerate their resettlement process [7]. Additionally, refugee resettlement, for many countries, is a regulatory process involving relocation from an asylum country to another country that has granted permanent settlement, and typically includes mandatory health screenings [1]. Therefore introducing research or other public health investigations into the 
regulatory process of resettlement could blur the perception of voluntary participation. For instance, this could occur if there is confusion between mandatory medical screenings (or associated required disease treatments for resettlement) and voluntary screenings (or voluntarry treatments) for investigative purposes. Similarly, there is also the risk of confusing investigations, particularly those with a tangible intervention, with humanitarian aid. Respecting the rights, values, and beliefs of individuals and communities, as well as ensuring privacy (particularly in refugee camps), safety, and autonomy are also critical when engaging refugees. These principles become even more important given acute survival needs (e.g., food, shelter, clean water), risks of re-traumatization, and stigmatization of certain conditions or situations in the surrounding community (e.g., mental health issues, sexual assault) $[3,4,6,7]$. Additional concerns arise in areas that may lack the capacity to provide appropriate technical guidance and oversight, such as in conflict settings [3].

The refugee context is ever-changing and therefore, the ethics principles followed and frameworks used to guide such investigations should be frequently examined and updated. Although a large body of literature exists outlining ethics principles and current debates in research ethics, there are few established refugee-specific ethics frameworks to guide such investigations, and no formal consensus about how basic research ethics principles should be interpreted in the refugee context. Additionally, while a handful of investigations have elicited data on thoughts and opinions about the investigation process and related ethical challenges directly from refugees [2], it is unclear how such responses and information have been translated to the activities of the wider scientific community $[2,14]$. We conducted a preliminary assessment of recent published literature to understand the application of ethics principles reported in investigations involving refugees in recent years and propose considerations and potential best practices to protect the welfare of refugees in research or investigation contexts.

\section{Methods}

\section{Information source, eligibility, and article selection}

We reviewed reports of refugee health-related investigations published in English from January 2015 to September 2018 available in PubMed. Search terms were kept broad to increase the likelihood of identifying relevant publications. The search terms used were "refugee" or "refugees" in the article title to capture articles for which refugees were the primary focus. To keep the number of publications for review manageable, the PubMed search was restricted to these keywords within the title. Additionally, although refugee populations are similar to asylees (and asylum seekers), internally displaced persons, and other migrants, their circumstances, regulatory processes of resettlement, and types of protections can differ. Therefore, we limited our search to only refugees to narrow the scope and conduct a more focused review (however, the inclusion of these populations in addition to refugees did not lead to exclusion of an article). Given that similar ethical procedures are followed for both research and non-research data collections, and many publications did not distinguish between the two, both types of investigations were included.

Upon establishing this initial list in PubMed, we conducted the second stage of article selection by thoroughly reviewing the title and abstract (and in some instances where the abstract lacked the required information, the full article) according to the following inclusion criteria: (a) subjects of the article were refugees (not aid workers, clinicians, etc.); (b) primary topic was health-related; and (c) investigators directly interacted with the refugees included in the analysis (primary data sources). The last criterion consequently excluded investigations that solely used surveillance data, refugee databases, or chart reviews (secondary data sources). Review articles, systematic reviews, meta-analyses, notes from the field, letters to the editor, methodological papers, and policy papers were also excluded.

\section{Measures}

After reviewing available literature on ethics in refugee investigations, the authors developed a data abstraction tool, with assistance from a refugee health expert and two public health ethics experts, to capture methodological aspects pertaining to ethics questions and dilemmas. We piloted the tool using 30 articles to be included in the analysis. As we reviewed those articles, we noted additional ethics-related scenarios raised or addressed, and we added a question to the data abstraction tool.

The final tool contained 64 variables (list of questions used can be found in 'Additional File 1'), including both quantitative and qualitative questions. Information collected included characteristics of each investigation (year published, journal type, investigator's home country, country in which the investigation was undertaken, type of institution, type of funding, investigation design, and primary health topic), as well as specific data points related to ethics considerations and methodological procedures. It was also understood that not all applications of ethics would be reported in the articles. However, due to the preliminary and exploratory nature of this analysis, we did not contact authors to obtain information not documented in the articles. If an author did not mention the information of interest, data abstractors filled in the variable as "not mentioned," rather than "no" to avoid potential misrepresentation of the investigation or 
misclassification of the data (this was particularly pertinent to the post-investigation variables).

\section{Data collection}

Four authors, abstracted data between July 1, 2018, and September 30, 2018. Each article was reviewed by one data abstractor.

After data abstraction was complete, $25 \%$ of each abstractor's articles were randomly selected for a second review by one of the other reviewers to check for data irregularities or mistakes. The two abstractors discussed any differences and reached a consensus. For the 25\% that underwent a second review, inter-reviewer agreement was high: average agreement rate per variable: 99.2\%, standard deviation: $1.7 \%$, range: 93.4 to $100 \%$; three quarters of the variables had $100 \%$ agreement. For the remaining $75 \%$ of the articles, each record was scanned for any missing data points or inaccuracies (e.g., two answers contradicting each other).

\section{Data Analysis}

Quantitative variables were analyzed using descriptive statistics. Qualitative variables were examined using a mixed methods approach including both an examination of the presence of a response (yes/mentioned vs. no/not mentioned) and an analysis of the response (identification of common themes).

\section{Results}

\section{Article selection}

Initial search results in PubMed yielded 912 articles, of which 288 (32\%) were included in the analysis following the second stage of selection (list of articles included can be found in 'Additional File 1'). Reasons for exclusion $(n=624)$ included: the article was a literature or systematic review, commentary, or meta-analysis (328/624, $53 \%)$; no interaction between investigators and participants $(135 / 624,22 \%)$; not health-related $(63 / 624,10 \%)$; refugees were not the subjects $(55 / 624,9 \%)$; and other (e.g., methodological, policy; 43/624, 7\%) (Fig. 1).

\section{Investigation characteristics}

Characteristics of the included investigations are shown in Tables 1 and 2 (and 'Additional File 1'). Most of the investigators were from the United States (90,31\%), Australia $(43,15 \%)$, or Germany $(16,6 \%)$ with most investigations conducted by academic institutions (212, 74\%). Funding was typically provided by a national governmental organization $(76,26 \%)$ or an academic institution (46, $16 \%)$. Approximately $33 \%(n=96)$ of investigations were conducted during displacement (68\% of these were in refugee camps), and most were cross-sectional by design (234, $81 \%)$. Nearly $42 \%$ used surveys or questionnaires for data collection; 33\% used interviews, and 9\% used focus groups, with similar distribution for both pre- and postresettlement. Common topics included mental health $(139,48 \%)$ and healthcare access (22, 8\%). Almost half (141, 49\%) included at least one special subpopulation (e.g., LGBTQ, pregnant women, people with disabilities, children under 5 years old, adults over 65 years old).

\section{Ethics applications \\ Protocol review and consent}

Ethics-related considerations and applications are presented in Tables 3 and 4. Approximately $94 \%$ of the included articles reported review of the investigation by an ethics committee. Two $(0.7 \%)$ stated no review was conducted: one was an audit, and therefore, according to the authors, did not meet the criteria for an ethics review; one was a student's thesis, and according to the authors, ethics approvals were neither required nor sought for student theses at their institution $[15,16]$. For 15 (5.2\%) articles, it was not stated whether an ethics committee reviewed. However, two of these 15 included language suggesting an ethics review (one discussed the protocol was reviewed by the "hospital administration," which could include an ethics board; one discussed that a review was conducted in a prior investigation with the same sample population, but it was unknown if the prior review accounted for procedures conducted in the included investigation) $[17,18]$.

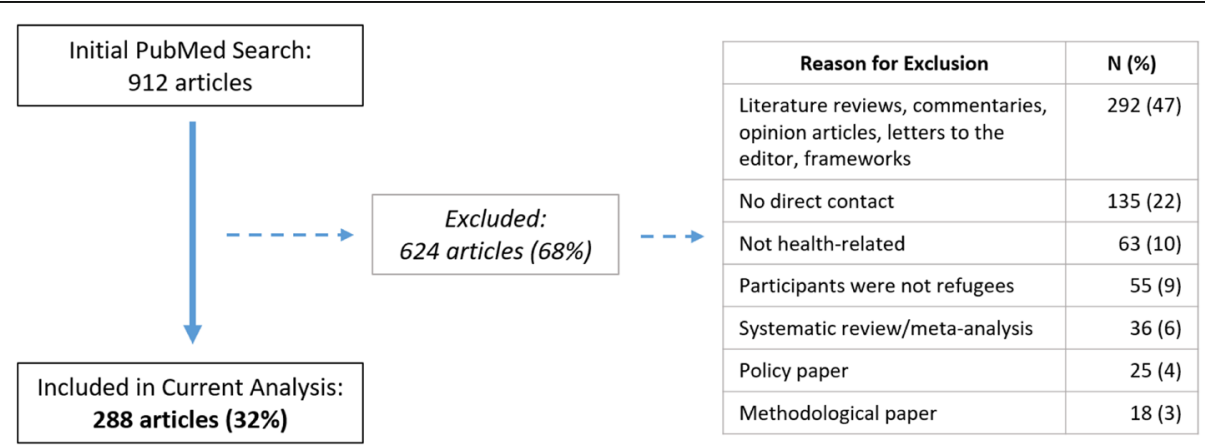

Fig. 1 Article selection for a review of ethics considerations in published refugee health literature 
Table 1 Characteristics of 288 articles in review of ethical considerations: author nationality, funding source, investigation design

\begin{tabular}{|c|c|}
\hline Characteristic & $\boldsymbol{N}(\%)$ \\
\hline \multicolumn{2}{|l|}{ Investigator's Home Country, top four and other } \\
\hline United States & $90(31.3)$ \\
\hline Australia & $43(14.9)$ \\
\hline Germany & $16(5.6)$ \\
\hline Canada & $15(5.2)$ \\
\hline Other & $124(43.1)$ \\
\hline \multicolumn{2}{|l|}{ Primary Institution } \\
\hline Academic institution & $212(73.6)$ \\
\hline Medical institution & $47(16.3)$ \\
\hline National government & $17(5.9)$ \\
\hline Nonprofit/nongovernmental organization & $7(2.4)$ \\
\hline State/local government & $2(0.7)$ \\
\hline Other & $3(1.0)$ \\
\hline \multicolumn{2}{|l|}{ Primary Funding Source } \\
\hline National government & $76(26.4)$ \\
\hline Academic institution & $46(16.0)$ \\
\hline Nonprofit/nongovernmental organization & $31(10.8)$ \\
\hline Medical institution & $21(7.3)$ \\
\hline Private & $14(4.9)$ \\
\hline State/local government & $4(1.4)$ \\
\hline Other & $13(4.5)$ \\
\hline None & $11(3.8)$ \\
\hline Unknown/not mentioned & $72(25.0)$ \\
\hline \multicolumn{2}{|l|}{ Investigation Design } \\
\hline Cross-sectional & $234(81.3)$ \\
\hline Prospective cohort & $29(10.1)$ \\
\hline Randomized control trial & $7(2.4)$ \\
\hline Other & $18(6.3)$ \\
\hline \multicolumn{2}{|l|}{ Investigation Type } \\
\hline Observational & $249(86.5)$ \\
\hline Intervention & $39(13.5)$ \\
\hline \multicolumn{2}{|l|}{ Time Point in Resettlement Process } \\
\hline Displaced, refugee camp & $64(22.2)$ \\
\hline Displaced, non-refugee camp (e.g., urban refugee) & $31(10.8)$ \\
\hline Post-resettlement & $192(66.7)$ \\
\hline Both, displaced and post-resettlement & $1(0.3)$ \\
\hline $\begin{array}{l}\text { Refugees Classified as Internally Displaced } \\
\text { (among those displaced in camp or non-camp } \\
\text { setting, } n=96 \text { ) }\end{array}$ & $7(7.3)$ \\
\hline $\begin{array}{l}\text { Investigation Conducted in Conflict Zone } \\
\text { (among displaced in camp or non-camp } \\
\text { setting, } n=96 \text { ) }\end{array}$ & $3(3.1)$ \\
\hline
\end{tabular}

For 264 (92\%) of the articles included, at least one of the investigators was from the country of the investigation. Of these 264, 248 (94\%) mentioned an ethics review (1\% none, $5 \%$ unknown). For $24(8 \%)$ of all of the articles included, the investigation team was comprised of only investigators from outside the country of investigation. Of these 24, 23 (96\%) mentioned an ethics review (4\% unknown), and of these 23: the authors of 11 (48\%) articles conducted both an internal (within the country of investigation) and external (within the investigators' home countries) ethics review, the authors of 4 (17\%) articles conducted an internal review only, and the authors of 7 (30\%) articles conducted an external review only. Finally, of the 288 articles included, only $8 \%(n=23)$ mentioned that their protocol was reviewed by representatives from the refugee community.

Obtaining consent was common (249, 87\%). About two-thirds $(162,65 \%)$ of the investigations who stated they obtained informed consent mentioned translating the consent. Additionally, approximately 68\% (197/288) mentioned the use of an interpreter at any point during the investigation. Only 7\% (of the 249 who obtained consent) discussed making consent an iterative process (obtaining consent at each stage of the investigation to ensure continued understanding of research procedures, the voluntariness of participation, and an understanding of what individuals are consenting for at each stage). Two investigations stated no consent was obtained, citing routine screening or clinical care and quality improvement as justifications for not obtaining consent $[19,20]$. Thirty-seven articles did not mention whether consent was obtained; of these, many were conducted as part of routine screening examinations at reception centers or during emergency or outbreak scenarios.

\section{Risk minimization}

Three-quarters of the investigations $(n=216)$ discussed methodological choices to minimize risks to participants (not mutually exclusive: $36 \%$ ensured cultural sensitivity; $28 \%$ trained the investigators before initiating the project; $15 \%$ ensured a doctor or counselor was available to participants in the event of physical or psychological distress; $12 \%$ conducted pilot investigations which allowed key community members to provide feedback on sensitive questions or best implementation strategies to ensure the safety, as well as mental and physical well-being, of refugees during the investigation). At least 20 articles stated that they engaged highly trained and qualified clinicians to administer the interventions and provided referrals as needed to culturally competent service providers [21-28].

\section{Privacy}

Overall, 50\% $(n=1543)$ mentioned undertaking privacy measures, which included conducting the investigation 
Table 2 Characteristics of 288 articles in review of ethical considerations: health topic, data source, participant demographics

\begin{tabular}{|c|c|}
\hline Characteristic & $\boldsymbol{N}(\%)$ \\
\hline \multicolumn{2}{|l|}{ Primary Health Topic } \\
\hline Mental health & $139(48.3)$ \\
\hline Access to healthcare & $22(7.6)$ \\
\hline General health profile & $21(7.3)$ \\
\hline Maternal and child health & $15(5.2)$ \\
\hline Nutrition and physical activity & $15(5.2)$ \\
\hline Injury and violence & $10(3.5)$ \\
\hline Other & $66(22.9)$ \\
\hline \multicolumn{2}{|l|}{ Primary Data Source } \\
\hline Surveys/questionnaires & $120(41.7)$ \\
\hline Individual interviews & $96(33.3)$ \\
\hline Focus groups & $27(9.4)$ \\
\hline Human specimen samples & $25(8.7)$ \\
\hline Medical records/programmatic databases ${ }^{1}$ & $12(4.2)$ \\
\hline Observations & $5(1.7)$ \\
\hline Other & $3(1.0)$ \\
\hline \multicolumn{2}{|l|}{ Age } \\
\hline$\geq 18$ years old & $160(55.6)$ \\
\hline$<18$ years old & $20(6.9)$ \\
\hline Both & $92(31.9)$ \\
\hline Unknown/not mentioned & $16(5.6)$ \\
\hline \multicolumn{2}{|l|}{ Special Populations (not mutually exclusive, $n=141$ ) } \\
\hline Over 65 years old & $77(26.7)$ \\
\hline Under 5 years old & $30(10.4)$ \\
\hline Pregnant women & $17(5.9)$ \\
\hline LGBTQ & $5(1.7)$ \\
\hline Disabled & $2(0.7)$ \\
\hline Other & $29(10.1)$ \\
\hline \multicolumn{2}{|l|}{ Sex } \\
\hline Females & $50(17.4)$ \\
\hline Males & $7(2.4)$ \\
\hline Both & $228(79.2)$ \\
\hline Unknown/not mentioned & $3(1.0)$ \\
\hline \multicolumn{2}{|c|}{ Pre-resettlement Country, top four and multiple countries } \\
\hline$>1$ country & $151(52.4)$ \\
\hline Syria & $25(8.7)$ \\
\hline Burma/Myanmar & $15(5.2)$ \\
\hline North Korea & $12(4.2)$ \\
\hline Iraq & $11(3.8)$ \\
\hline Other & $74(25.7)$ \\
\hline
\end{tabular}

Post-resettlement Country, top four and other ( $n=193$ that included refugees that has resettled after displacement period)

United States
Table 2 Characteristics of 288 articles in review of ethical considerations: health topic, data source, participant demographics (Continued)

\begin{tabular}{lc}
\hline Characteristic & $\boldsymbol{N}(\%)$ \\
\hline Australia & $32(16.6)$ \\
Germany & $15(7.8)$ \\
Canada & $12(6.2)$ \\
Other & $70(36.3)$ \\
\hline${ }^{1}$ Secondary data source used involved direct interaction with refugees in \\
other parts of the investigation, qualifying the investigation for inclusion
\end{tabular}

on a sensitive topic in a school instead of a health clinic (i.e., a more neutral location) to prevent non-participants from associating the participants with the sensitive health topic and maintain the anonymity of the participants [27] and providing the opportunity to decline voice recording [29-31]. Private data collection locations were used in $64 \%(n=183)$ of investigations $(63 \%$ post-resettlement, $66 \%$ during displacement), including some in which participants were allowed to choose the location. Some investigations used homogenous focus groups based on sex or other demographic characteristics to create a safe space to protect participant identities [32, 33]; one investigation allowed individuals to opt for an individual interview over a focus group [34]. Of the 197 investigations with interpreters, $46(23 \%)$ used native speakers from the community of interest. Finally, $19 \%(n=55)$ of the articles stated that data were stored without identifiers, and many others indicated that data were stored securely using encrypted software, locked cabinet files, or secure servers.

\section{Incentives (reimbursements)}

Providing incentives, also referred to as reimbursements, was uncommon $(66,23 \%)$. Incentives were either nonmonetary (11\%), such as clothing or food, or monetary (89\%). When monetary incentives were used, approximately one-fifth (20\%) exceeded 30 USD, $61 \%$ were less than or equal to 30 USD, and 19\% did not state the amount provided. Non-monetary or small monetary incentives $(<5$ USD) were typically provided inside refugee camps, whereas larger monetary incentives were provided post-resettlement.

\section{Transparency, trust, engagement, and respect}

Approximately $57 \%$ of authors discussed measures undertaken to ensure transparency of the investigation within the community, including holding pre-investigation informational meetings; and 40\% undertook measures to establish participant and community trust, such as working through trusted organizations or community leaders. Most authors (91\% of all articles included, $n=262$ ) discussed community stakeholder involvement, with common stakeholders being health clinics or hospitals, community members, and local nongovernmental organizations. Community members 
Table 3 Ethics considerations: ethics review, consent, risk minimization, privacy

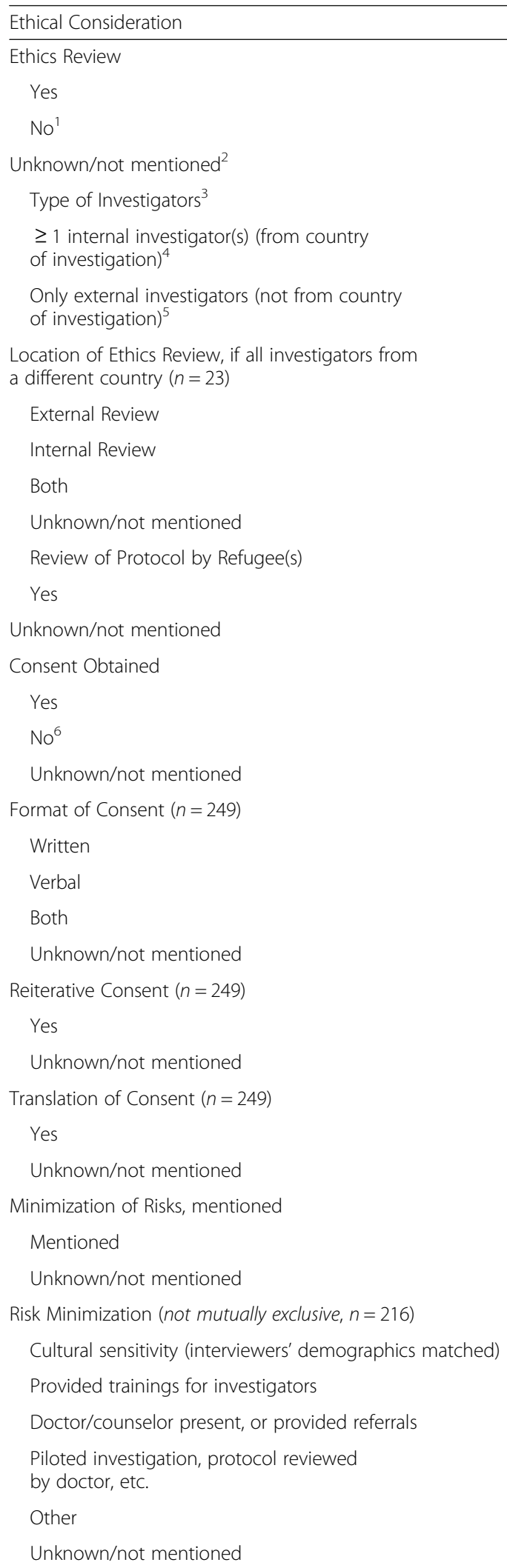

$\boldsymbol{N}(\%)$

271 (94.1)

$2(0.7)$

$15(5.2)$

$264(91.7)$

$24(8.3)$

7 (30.4)

$4(17.4)$

$11(47.8)$

$1(4.3)$

$23(8.0)$

265 (92)

249 (86.5)

$2(0.7)$

37 (12.8)

116 (46.6)

38 (15.3)

$27(10.8)$

68 (27.3)

$17(6.8)$

232 (93.2)

$162(65.1)$

87 (34.9)

216 (75.0)

$72(25.0)$

$78(36.1)$

61 (28.2)

33 (15.3)

25 (11.6)

109 (50.5)

$72(25.0)$
Table 3 Ethics considerations: ethics review, consent, risk minimization, privacy (Continued)

\begin{tabular}{ll}
\hline Ethical Consideration & $\boldsymbol{N}(\%)$ \\
\hline Mentioned & $143(49.7)$ \\
Unknown/not mentioned & $145(50.3)$ \\
Location of Data Collection & \\
Private & $183(63.5)$ \\
Public & $24(8.3)$ \\
Unknown/not mentioned & $81(28.1)$ \\
Data Stored as Deidentified & \\
Yes & $55(19.1)$ \\
Unknown/not mentioned & $233(80.9)$ \\
Collection of Identifiable Information & \\
Yes & $66(22.9)$ \\
No & $47(16.3)$ \\
Unknown/not mentioned & $175(60.8)$
\end{tabular}

Use of an Interpreter

Yes

Sometimes/when available $6(2.1)$

No

$15(5.2)$

Unknown/not mentioned

$76(26.4)$

Source of Interpreter $(n=197)$

Native speakers, from community

$46(23.4)$

Native speakers, from another community

$19(9.6)$

Native speakers, unspecified community

$17(8.6)$

Non-native speakers

$6(3.0)$

Unknown/not mentioned

$109(55.3)$

Digital/Audio Recording of Any Portion of Data Collection

Yes

No

Unknown/not mentioned

$153(53.1)$

${ }^{1}$ No ethics reviews: (1) audit that did not meet the criteria for an ethics review, (2) an ethics review was not required/sought for student thesis $[15,16]$

${ }^{2}$ Two of the unknown ethics reviews could have received an ethics review: (1) stated approval by hospital administration, (2) stated that prior study with same sample population had an ethics review $[17,18]$

${ }^{3}$ Using the list of authors

${ }^{4} 248$ (94\%) mentioned an ethics review, 14 (5\%) unknown, 2 (1\%) no review

${ }^{5} 23(96 \%)$ mentioned an ethics review, 1 (4\%) unknown

"Two investigations stated "no" consent was obtained, citing routine

screening/clinical care and quality improvement as justifications [19, 20]

helped to recruit participants in $50 \%(n=145)$ of the investigations. Cultural perspectives were considered in $42 \%$ ( $n=121)$ of the investigations and included gender and religious norms such as matching the gender of the interviewer and/or interpreter to that of the participant, as well as ensuring homogenous focus groups (e.g., all female).

\section{Post-investigation support}

After the investigations, 9\% $(n=25)$ of all of the 288 articles included presented results to participants,

Privacy Measures Undertaken 
Table 4 Ethical considerations: incentive use, establishing trust, transparency, benefits, results sharing

\begin{tabular}{l} 
Ethical Consideration \\
\hline Use of Incentives \\
Yes \\
Unknown/not mentioned \\
Investigator(s) Transparency' \\
Mentioned \\
Unknown/not mentioned \\
Establishment of Trust by Investigators ${ }^{2}$ \\
Yes \\
Unknown/not mentioned \\
Stakeholder Engagement (not mutually exclusive, $\mathrm{n}=262)^{3}$ \\
Health clinics/hospitals \\
Community members \\
Local nonprofit/nongovernmental organization \\
Community leaders/elders \\
National government \\
Local government \\
Local private business \\
Other \\
Unknown/not mentioned \\
Community Assisted with Recruitment \\
Yes \\
Unknown/not mentioned
\end{tabular}

Cultural Practices Considered (not mutually exclusive, $\mathrm{n}=121$ )

Gender norms
Permission to conduct
Social hierarchy/order
Age hierarchy
Religious norms
Other

Results Presented to Participants

Yes

Unknown/not mentioned

Results Presented to Community

Yes

Unknown/not mentioned

Social Justice/Health Equity Considered

Mentioned

Unknown/not mentioned

Community Empowerment

Trainings

Community education
Table 4 Ethical considerations: incentive use, establishing trust, transparency, benefits, results sharing (Continued)

\begin{tabular}{ll}
\hline Ethical Consideration & $\mathrm{N}(\%)$ \\
\hline Provided a voice & $2(0.7)$ \\
Unknown/not mentioned & $233(80.9)$ \\
Intervention Provided to Larger Community $(n=39)$ & \\
Yes & $5(12.8)$ \\
Unknown/not mentioned & $34(87.2)$ \\
Support of Intervention Post-investigation & \\
Yes & $35(12.2)$ \\
Unknown/not mentioned & $253(87.8)$
\end{tabular}

Community Provided with Resources to Continue Intervention Yes

Unknown/not mentioned

${ }^{1}$ Of those who mentioned transparency (not mutually exclusive): 58 (41.1\%) held pre-investigation meetings, 54 (38.3\%) ensured translation of consent/ materials, 50 (35.5\%) explicitly explained participation was voluntary ${ }^{2}$ Of those who mentioned establishing trust (not mutually exclusive): 67 (58.8\%) worked through community partners, $23(20.2 \%)$ built relationships with community before investigation, $20(17.5 \%)$ took actions to respect cultural norms

${ }^{3}$ Type of stakeholder engagement: 144 (50.0\%) recruitment, 36 (12.5\%) data collection, 25 (8.7\%) funding, 7 (2.4\%) investigation design, 103 (35.8\%) multiple of the previously mentioned types of engagement, $5(1.7 \%)$ other forms of engagement

and 3\% $(n=9)$ of all of the articles included presented results to the community. Approximately 13\% mentioned post-investigation support to the participants or community (57\% of these were postresettlement investigations), and $13 \%$ of the 39 interventional investigations provided the intervention to the wider community after the investigation concluded. Discussion of community empowerment was more common for investigations conducted during the displacement period (30\%) than after formal resettlement (14\%). Twenty-one (7\%) investigations empowered the community through trainings for community workers (healthcare workers, refugees hired and trained for the investigation, etc.), and 20 (7\%) investigations provided health education to the community. Concerns of health equity and social justice were addressed by some (e.g., providing counseling or treatment when needed even when treatment was not part of the investigation [35], and providing vaccinations for all members in the community regardless of participation [36]). Given the explorative nature of most of the investigations included, sustainability of interventions was limited after project completion, with many investigators bringing screening equipment or other supplies for use only during data collection. However, in at least one case, resources were available to continue health programs or provide interventions to participants or the community beyond the investigation [37]. 


\section{Discussion}

Although relatively limited in scope, our analysis provided an opportunity to describe the application of research ethics principles in refugee settings cited within recently published articles. With the growing numbers of displaced populations worldwide and the ever-changing context for causes of displacement, there continues to be a need for conducting public health research among refugee populations by diverse groups including governments, academic institutions, and non-governmental organizations. This assessment sets the stage for a more in-depth and comprehensive look at ethics concepts and their applications.

From our assessment, we found that the extent to which ethics principles were reported varied greatly across the refugee health literature we examined. Our findings highlight the need for a current understanding of ethics and the application of ethics principles in refugee health investigations. Moreover, there is still likely room for improvements to the investigation and review processes regarding ethics within the field of refugee health research. Additionally, the refugee context changes over time, as does critical thinking on research best practices, highlighting the need to repeat such analyses periodically to ensure research practices as they relate to refugees evolve accordingly. Although our current analysis provides important information regarding the present context and circumstances of refugees, the implementation of ethics principles in refugee health research should be revisited along with the changing landscape.
For the purposes of our discussion, we divided the investigational process into three phases-pre-investigation, investigation, and post-investigation-to discuss ethical challenges and potential best practices the investigator can undertake. However, many of these considerations span the full investigation process. We acknowledge best practices will vary by context, setting, and investigation characteristics. A summary of these proposed best practices described can be found in Table 5.

\section{Pre-investigation phase}

To ensure investigations are conducted with respect for and protection of the participating individuals and community from the onset, ethical considerations should be at the forefront throughout the pre-investigation phase, during which questions are refined and methodological details are decided (e.g., location of investigation, content and logistics of consent, appropriate ethical and community approvals, mechanisms for recruitment of participants).

Early community and stakeholder engagement is critical during the pre-investigation phase. Such engagement can help to bridge cultural differences and establish trust within the community, as well as provide an outlet for the community to voice their concerns and thoughts [9, 38]. For instance, this engagement can take the form of a formal community needs assessment or informal community-wide meetings to identify the type(s) of services needed or the benefits and risks of a specific

Table $\mathbf{5}$ List of some potential best practices ${ }^{1}$ to consider when conducting health-related investigation within refugee populations ${ }^{2}$

\begin{tabular}{|c|c|c|}
\hline Pre-investigation Phase & Investigation Phase & Post-investigation Phase \\
\hline \multicolumn{3}{|c|}{$\begin{array}{l}\text { - Ensure early engagement with key community leaders, stakeholders, and overall community (pre-investigation meetings, etc.) } \\
\text { to ensure transparency and trust; and continue throughout investigation }\end{array}$} \\
\hline $\begin{array}{l}\text { - Conduct a pilot investigation to allow key } \\
\text { community members to provide feedback on } \\
\text { sensitive questions and implementation strategies } \\
\text { - Ensure review of protocol by an ethics committee in } \\
\text { the countries of both the investigator(s) and the } \\
\text { investigation, and by members of the refugee } \\
\text { community, to minimize risks } \\
\text { - Address potential power imbalances that may affect } \\
\text { the investigation or who is represented } \\
\text { - Prevent over-researching by searching literature be- } \\
\text { fore investigating } \\
\text { - Train investigators (e.g., in cultural competency) } \\
\text { - Differentiate investigation activities from social } \\
\text { services } \\
\text { - Inform community members on the purpose of the } \\
\text { investigation } \\
\text { - Give a voice to the community and key stakeholders } \\
\text { to comment on the potential investigation and ask } \\
\text { questions }\end{array}$ & $\begin{array}{l}\text { - Ensure a private location for data collection (hard } \\
\text { to find in refugee camps) } \\
\text { - Carefully consider the risks to privacy when using } \\
\text { interpreters from the community, and consider } \\
\text { hiring interpreters from outside the community } \\
\text { - Carefully consider the risks to privacy when } \\
\text { conducting focus groups (consider separating by } \\
\text { gender, age, or religion if appropriate) } \\
\text { - If an incentive is used, place its value in context } \\
\text { - Consider iterative consent } \\
\text { - Minimize risks and harm (e.g., ensure a doctor/ } \\
\text { counselor is available in the event of physical or } \\
\text { psychological distress) } \\
\text { - Educate individuals on their rights as potential } \\
\text { participants before they provide their consent } \\
\text { - Ensure participation does not interfere with access } \\
\text { to services } \\
\text { - Ensure informed consent procedure is sensitive to } \\
\text { cultural practices and norms, and practical for } \\
\text { populations that have low literacy or little } \\
\text { understanding of the investigation process } \\
\text { - Present preliminary results to stakeholders to } \\
\text { improve interpretation of results }\end{array}$ & $\begin{array}{l}\text { - Present final results to both } \\
\text { participants and their community } \\
\text { - If an intervention is provided } \\
\text { engage with the community and } \\
\text { stakeholders to ensure its } \\
\text { sustainability } \\
\text { - Provide community members with } \\
\text { job skills to be used post- } \\
\text { investigation } \\
\text { - Empower community health } \\
\text { workers through trainings } \\
\text { - Provide continued and sustainable } \\
\text { health educational classes for the } \\
\text { community } \\
\text { - Allow participants and community } \\
\text { members to comment on the } \\
\text { results } \\
\text { - Identify ways to provide immediate } \\
\text { benefits in addition to long-term, } \\
\text { sustainable ones }\end{array}$ \\
\hline
\end{tabular}

\footnotetext{
${ }^{1}$ Best practices, and the weight awarded to each practice, should and will vary by context, setting, and investigation characteristics; not an exhaustive list

${ }^{2}$ And to consider discussing these considerations in published literature, as they are able
} 
project or intervention. Pre-investigation meetings also enable investigators to fully communicate to community members how and why an investigation is being conducted, and the potential benefits for the community. Feedback from the community through key informant interviews or focus groups can also shape investigation protocols and procedures to add cultural competence. For example, the content and format of consent can meet participants' information needs, or location of interviews can show respect for the community [9]. Transparency during this phase can address concerns regarding the impact on the community and answer questions for those unfamiliar with the investigational process [6]. Transparency and open communication with the community also ensure the investigation addresses information gaps in addition to the priorities and interests of the investigator [11].

Furthermore, in conflict settings where investigators need security guards, community engagement is particularly important to address potential power imbalances. For instance, in some cases, armed guards or other authority figures may accompany investigators to ensure their safety. Such non-routine security measures can unintentionally produce undue fear and the perception of coercion, particularly among refugees previously mistreated by authority figures. Therefore, prior community engagement may be needed to establish trust and ameliorate these feelings [5, 39].

Additionally, during community engagement and project development, investigators should (a) clearly differentiate investigational activities from provision of social services or humanitarian aid, or processes required for resettlement and (b) ensure that participation does not affect provision of services (e.g., by scheduling interviews outside ration distribution hours, or ensuring the investigation does not take medical personnel away from routine duties) $[6,11,13]$. Investigations differ from service provision in that the success of an intervention used in an investigation is still unproven. Additionally, often only a small portion of the eligible population is recruited to participate in an investigation. Yet for those unfamiliar with investigations, these distinctions can be confusing and appear unfair, as when only a small portion of the population receives investigational hygiene products or nutritional supplements. It is also essential that participant recruitment and selection strategies are unbiased to ensure all eligible individuals or groups have an equal opportunity to participate. When some people are excluded, transparency is needed to adequately explain this differentiation to the entire community, and to convey that the goal of the analysis is to provide a benefit for everyone in the future [11, 40].

Overall best practices for community engagement include pre-investigation meetings with community leaders and members [6]. Such leaders can often be identified through UNHCR and nongovernmental organizations in the refugee camp or community where the investigation is to be held. However, investigators must anticipate that the power hierarchy within the refugee population might unduly influence potential participants or predetermine which individuals or groups have access to the research, and take steps to minimize such influences [7, 41]. It is also important to note that although community engagement is initiated pre-investigation, significant benefit comes from its continuation throughout the investigation.

Another key step during the pre-investigation phase is an ethics review of the investigation protocol. It was promising to see that the majority of investigations mentioned review by a formal ethics committee; however, not all discussed completion of this review in both the investigators' home country and the country where the investigation took place. Some investigators' ethnic and cultural backgrounds differed from those of the participants, setting the stage for potential clashes between cultural, gender, or religious norms [42]. An ethics review within the country of investigation helps to ensure methodologies align with community practices. It also minimizes risks not often identified by an ethics committee unfamiliar with the local culture [38, 43].

However, even ethics committee members inside the country of investigation may be unfamiliar with refugee needs, circumstances, and vulnerabilities and therefore not fully able to represent the interests of refugees displaced from another country. Thus additional review by members of the refugee community in which the investigation is occurring may prove most insightful. Few articles mentioned the presence of a refugee on the committee or review of the protocol by refugees. Such reviewers are often better positioned to weigh the risks and judge the value of the investigation and determine the cultural feasibility and appropriateness of the methods [13]. These reviewers may also play the role of ethics committees in locations that lack official ethics committees, such as conflict settings.

Finally, investigators should carefully consider the need for and utility of their investigation before initiation to prevent overburdening refugees with similar or repetitive investigations, often referred to as "overresearching" [3, 6, 7, 38]. In our review, we were unable to assess the degree to which investigators strove to prevent over-researching. The risks of over-researching are particularly relevant in mental health investigations, which may pose a risk of re-traumatization. All investigations should have both practical and actionable outcomes that would not be possible without the investigation $[6,13]$. Best practices to prevent overresearching might include thorough literature reviews and consultations with experts in the subject area to 
understand whether a proposed investigation is truly necessary to develop effective interventions. Another could be stricter requirements by ethics review committees that investigators demonstrate that their proposed investigations are truly warranted and meet the "reasonable person standard," which requires investigators to assess the need for a particular investigation to avoid duplication and excess burden $[44,45]$. The timing of the investigation may also affect the degree of burden on the target population. For instance, resettled refugees may feel less burdened by investigations because many of their acute needs have been addressed, while those not yet resettled may be more vulnerable. Overall, the investigation's impact should be carefully considered to ensure the findings can effectively benefit the population; and especially in emergency response settings, new investigations should be initiated only when necessary data cannot be obtained in other ways [11].

\section{Investigation phase}

Ensuring ethical implementation during the investigation encompasses the overarching principles of minimizing stigma and other harm, ensuring privacy and respect, and preventing undue influence or coercion. A majority of the investigations in our analysis ensured a private location for data collection, with some investigations only collecting de-identified data or choosing individual interviews over focus groups (although held in private locations, the latter are not truly private) [7, 38]. Nonetheless, finding a private location can be challenging in refugee camps, and even if the information shared by participants is kept private, it may be difficult to keep participants' names confidential. Participation may involve disclosing characteristics that could increase risks of stigma or place an individual in danger if revealed to other community members (e.g., rape, sexual identity, mental illness, sexually transmitted infections) $[5,7]$. Additionally, many of the articles included in our analysis mentioned the use of interpreters from the community, which can also affect privacy. Although at the surface level these individuals appear to be the most appropriate cultural brokers, they can also (a) bias the results in that, fearing stigma, the participant declines to share information, or, potentially worse, (b) spread sensitive and private information disclosed by the participant into the community $[5,38,39]$. To mitigate these risks, one could seek community input to assess the acceptability of using interpreters (which could vary by health topic and setting) from the community, and if deemed unacceptable, hire bilingual individuals from outside the community when possible. Ultimately, strict privacy and confidentiality measures help to prevent further emotional or physical harm. As in the pre-investigation phase, cultural practices should be considered when choosing methods for ensuring privacy and confidentiality [38]. If focus groups are considered necessary for sensitive investigations, optimal standards could include separating the groups by gender, age, religion, or other factors, if deemed appropriate $[9,13,38,39]$.

Investigators also need to be knowledgeable about the refugee experience to prevent undue influence [5]. For instance, in our review, incentive type and value ranged greatly. Use of incentives needs to be placed in context to understand the amount of influence they may have. Five USD inside a refugee camp in Uganda, for instance, can have a drastically higher value than the same incentive provided after resettlement in the United States. Additionally, if implemented inappropriately, incentives can contribute to inequality between participants and non-participants. Engagement with community leaders, local nongovernmental organizations, or other refugee health experts may help in establishing whether incentives have appropriate values or whether they should be offered at all.

The complexities surrounding meaningful informed consent also require attention [5, 38]. Many refugees have never participated in investigations before and may not understand the concept of research or the investigation process [13]. For example, in a previous assessment of refugee knowledge around scientific investigations, less than half (44\%) of refugee respondents correctly answered "false" when asked whether "once somebody starts participating they are not allowed to quit" [46]. Although this percentage may have been influenced by communication barriers, it indicates that informed consent may not always be communicated effectively to refugee participants.

Our review of the literature reveals promising adherence to basic ethics principles for obtaining consent, a cornerstone to protecting participants' autonomy in all public health and biomedical investigations [47]. However, one should also consider the format and type of consent to ensure complete understanding and respect. Most of the investigations we reviewed obtained written consent, but not all addressed language barriers, literacy levels, languages that do not have a true written form, and cultural normalcy (e.g., in some cultures, verbal consent holds a higher value) [13]. Translation of consent into the language the participant prefers may minimize misinterpretations. Additionally, although written consent remains the norm, it can prove challenging in refugee investigations. Options for non-written consent may include verbal consent, audio or video-recorded consent, or witnessed consent (a witness signs) [48]. Innovative methods such as videos, illustrations, or other visual aids to help explain and obtain consent may also prove useful [48]. Few articles mentioned the use of iterative consent, a process by which individuals consent at each phase of 
the investigation. Such iterations remind refugees about the research procedures and that their participation is voluntary, which can help reduce feelings of pressure to complete the investigation [5]. Additionally, in the event that human biologic specimens are collected, ownership of these specimens should be discussed with respect for cultural and religious beliefs about the human body during the consent process. Ultimately, informed consent must be sensitive to cultural practices and norms, as well as practical for populations that have low literacy rates or limited understanding of the research and investigation process to help ensure true autonomy $[3,6]$.

\section{Post-investigation phase}

Finally, although often overlooked, the application of ethical practices should not cease after the investigation has ended. Few articles discussed ethical considerations for this phase of investigations, but such considerations are not always reported in published articles and can occur after publication. Nonetheless, post-investigation activities include aspects of social justice and health equity that should not be ignored [40, 49]. Best practices post-investigation may include empowering community health workers through trainings during or after the investigation; employing local refugees to help with the investigation, thereby providing them with job skills that will be useful post-investigation (this would often happen in the pre-investigation stage with the benefits extending into post-investigation); providing the means to sustain health education classes for the community; and providing resources to continue an effective intervention (or if applicable, providing the intervention to the wider community) $[6,40,50]$. Given the transient nature of refugee populations, the timing of such reciprocity is key. Sometimes participants move before investigation results are translated into permanent interventions; therefore, investigators should identify ways to provide immediate benefits to participants who may not see the investigation's long-term impact [7, 49]. Lastly, it is important to consider ensuring "reasonable availability" of an intervention that is demonstrated to be effective (i.e., ensuring the intervention is available free or at a reasonable cost to the community in which it was tested) [50].

Transparency after the analysis is also key and includes presenting the findings to both the participants and the wider refugee community [50]. Power imbalances can be addressed here by allowing individuals to comment on the results and their interpretation rather than merely hear the investigators' final framework or conclusions [6]. This, as well as other best practices outlined above, follow the community-based participatory research approach (an approach that collaboratively includes all parties involved throughout the research process, acknowledging that each party brings unique strengths and perspectives) [51].
Ultimately, many of the basic principles discussed above should also be applied in non-refugee public health investigations. Nonetheless, refugees as a population have unique vulnerabilities that warrant a greater level of diligence in avoiding research-related harm. For instance, refugees often differ from non-refugees in the degree of political protections or types of social services offered to them. Therefore, the manner in which these principles are applied, and the emphasis given to certain principles, should be specific to the refugees' individual circumstances. The addition of requirements by funding agencies and journals to outline and document procedures used may also help to ensure adherence to ethical guidelines.

\section{Limitations}

Our analysis was subject to several limitations. Given that not all journals require publication of all ethics applications we assessed, and publication requirements (word count, structure, reporting of post-investigation activities, etc.) vary, ethics considerations may have been omitted from the published manuscripts. As our analysis only accounted for actions reported within the published literature, we acknowledge our results are likely an underestimation, particularly of post-investigation activities. Therefore, we have drawn conclusions only in regard to the procedures and protocols investigators reported, and argue for the importance of increased reporting of ethics applications. It is also important to acknowledge that differing approval processes between research and non-research investigations may have affected the application of ethical standards. Additionally, the use of only one publication database and the inclusion of only articles in English language may have limited the types of articles included (e.g., social science articles may have been excluded). Furthermore, restricting the time of publication to 2015 through 2018 prevented the ability to analyze how adherence to reported ethical protocols evolved or varied over time. The analysis was also restricted to those with the words "refugee" or "refugees" in the title, which may have excluded some articles that did not mention the study population in the title but would have otherwise met the inclusion criteria. Additionally, this analysis was specific to refugees and the search criteria excluded other migrant study populations, such as asylees (when the article was solely focused on non-refugee migrants; some articles included both refugees and other migrants, and these papers were included in the current analysis). Although many of the basic ethics principles examined in our analysis translate to other migrant populations, there are also differences in the regulatory processes of resettlement that makes these groups different and warrant a deeper assessment of ethics principles for different 
migrant groups beyond the scope of this analysis. Future in-depth assessments should focus on these populations. Data collection errors might have been reduced if resources had been available to conduct a second review of all articles. However, given the high agreement rate on articles for which we did a second review, the number of data collection errors is likely low. Finally, due to the narrow scope of our analysis, we limited inclusion to primary data collections. Nonetheless, ethical dilemmas can arise in secondary data analyses. For instance, many government agencies and nongovernmental organizations collect program data intended for internal use that are later used by investigators. Such scenarios should be explored in future reviews.

\section{Recommendations}

Refugee investigations are needed. Investigators, in addition to maintaining the highest level of scientific integrity and rigor, have a duty to act ethically. The investigators' responsibility extends beyond securing an ethics committee approval, especially given that refugees are not always included as a "vulnerable population" (populations identified in need of enhanced protections), to ensuring protection of the welfare and dignity of participants (and documentation of that protection) [11]. For instance, the US human subjects research regulations (federal policy for protection of human research subjects, which defines the processes for ethics review and approval) does not include refugees as a vulnerable population [11]. Yet the field of ethics is dynamic, and often difficult to navigate. Ethics is far from an exact science, and emphasis on specific considerations and applications varies with population and setting. Our objective was to describe the breadth of application of ethics principles and identify possible gaps in their implementation, potentially stemming from the lack of a guiding research ethics framework in the field of refugee health.

There exists a need for an updated and comprehensive refugee-specific ethics framework to guide future investigations. This framework should include the basic principles found in both the Belmont Report and current public health frameworks, as well as other philosophies, including ethical guidance regarding underserved communities and ethics of community practice $[11,49,52-$ 54]. It would also require a degree of specificity to refugee populations that accounts for their heightened vulnerability and the characteristics that contribute to this vulnerability that make refugees unlike other individuals (e.g., limited political protections of stateless individuals, living conditions inside refugee camps, prior torture or trauma, economic instability, population mobility). Such a framework requires flexibility in order to remain applicable to the diversity in age, socioeconomic status, education level, and cultural practices among refugee populations, meaning that the weight given to a particular principle can and often should vary by context [49]. For instance, privacy should be held to the highest of standards when interviewing LGBTQ youth in a refugee camp, where disclosure of sexual orientation could place the individual in danger; but privacy may not take priority when examining less-stigmatized topics [49]. An established framework that identifies innovative solutions to protect refugees while ensuring scientific validity would aid in improving future investigations. Furthermore, the principles outlined in the framework could provide guidance and context to assist ethics committee reviewers in assessing the ethical integrity of proposals, particularly for reviewers unfamiliar with refugee health.

The best practices we identified both in our literature review and in developing the methodology for our literature review may help lead to developing a framework. To provide a supporting structure for such a framework, we propose three key areas: engage, educate, and empower.

\section{Engage}

Engage with stakeholders, community leaders, and community members before and throughout the investigation. This engagement helps to create trust, transparency, and collaboration. Additionally, early engagement with ethics committees helps to minimize risks of adverse outcomes.

\section{Educate}

Educate investigators (interviewers, data collectors, analysts, etc.) on topics such as cultural competency and ethics. Educate community members on their rights as potential participants in the investigation before you ask for their consent. Ethics committee reviewers may also benefit from education to ensure they are aware of the complex vulnerabilities of refugee populations.

\section{Empower}

Empower participants by ensuring they understand that participation is voluntary. Empower the community post-investigation by presenting results at forums (where community members can comment), ensuring sustainability of an intervention that the community can assume ownership of, or providing classes or trainings.

\section{Conclusion}

Overall, we found that ethics information is not always systematically reported in current published literature reporting on investigations involving refugees. Publications often did not document ethical considerations, and therefore, we encountered difficulties discerning which principles were followed. We did find two key points: (1) review of the investigation protocols by refugees themselves was reported infrequently (only 8\%), and (2) postinvestigation support or engagement for both the 
participants and their community was reported minimally (although the authors recognize the potential for omission of these in manuscripts submitted for publication, if not main focus of the investigation).

In conclusion, our analysis identified a number of complex ethical challenges in conducting refugee healthrelated investigations and found evidence of room for improvement in adherence to ethics principles and their documentation in resulting publications. Most importantly, we have described the unique characteristics of refugee populations that suggest a need for greater emphasis on particular ethics principles and warrant the development of a refugee-specific ethics framework to aid investigators in the field. Scientifically valid investigations with ethically collected data provide the foundation for policy and interventions, and therefore, investigators should make the fullest effort to ensure respect and safety for refugee participants and their communities.

\section{Supplementary information}

Supplementary information accompanies this paper at https://doi.org/10. 1186/s13031-020-00283-Z.

Additional file 1. Articles included in review and additional characteristics. Additional file $1^{\prime}$ includes the list of the 288 articles used in the review of ethical considerations in refugee health literature, additional characteristics of these 288 articles, and a list of the 64 variables abstracted from each article.

\section{Abbreviations}

UNHCR: UN Refugee Agency; LGBTQ: Lesbian, gay, bisexual, transgender, queer; USD: United States dollar

\section{Authors' contributions}

NM conceived the project idea. ES developed the tool for data collection, conducted data collection, conducted the analysis, and was a major contributor to writing the manuscript. AD, JW, and PS contributed to the refining of the data collection tool, data collection, and manuscript development/revision. NM, NC, DB, and LO contributed to the study design and development of the data abstraction tool, provided insights into final conclusions, contributed to manuscript development/revisions, and served as subject matter experts. The author(s) read and approved the final manuscript.

\section{Authors' information}

ES is supported via an appointment to the Applied Epidemiology Fellowship Program administered by the Council of State and Territorial Epidemiologists (CSTE) and funded by the Centers for Disease Control and Prevention (CDC) Cooperative Agreement Number 1U380T000143-05.

\section{Funding}

This report was supported in part by an appointment (ES) to the Applied Epidemiology Fellowship Program administered by the Council of State and Territorial Epidemiologists (CSTE) and funded by the Centers for Disease Control and Prevention (CDC) Cooperative Agreement Number 1U380T000143-05

\section{Availability of data and materials}

The datasets used and/or analyzed during the current study are available from the corresponding author on reasonable request.

Ethics approval and consent to participate

Not applicable.
Consent for publication

All authors have provided consent for publication.

\section{Competing interests}

The authors declare that they have no competing interests.

\section{Author details}

'Division of Global Migration and Quarantine, Centers for Disease Control and Prevention, 1600 Clifton Road, MS EO3, Atlanta, GA 30333, USA. ²CD/ CSTE Applied Epidemiology Fellowship Program, Atlanta, Georgia, USA. ${ }^{3}$ Oak Ridge Institute for Science and Education, Oak Ridge, Tennessee, USA. ${ }^{4}$ Eagle Global Scientific, LLC, Atlanta, Georgia, USA. ${ }^{5}$ Office of Scientific Integrity, Office of Science, Centers for Disease Control and Prevention, Atlanta, GA, USA.

Received: 6 December 2019 Accepted: 2 June 2020

Published online: 20 June 2020

\section{References}

1. UNHCR. Global Trends Forced Displacement in 2018. 2018. https://www. unhcr.org/globaltrends2018/. Accessed 19 April 2019.

2. Gabriel P, Kaczorowski J, Berry N. Recruitment of refugees for health research: a qualitative study to add refugees' perspectives. Int J Environ Res Public Health. 2017;14(2):125

3. Ford N, Mills EJ, Zachariah R, Upshur R. Ethics of conducting research in conflict settings. Confl Health. 2009;3:7.

4. Leaning J. Ethics of research in refugee populations. Lancet. 2001;357(9266): 1432-3

5. Mackenzie C, McDowell C, Pittaway E. Beyond 'do no harm': the challenge of constructing ethical relationships in refugee research. J Refug Stud. 2007; 20(2):299-319.

6. Tilbury F. Ethical dilemmas: principles and practice in research with African refugees. Monash Bioeth Rev. 2006;25(1):75-84.

7. Zwi AB, Grove NJ, Mackenzie C, Pittaway E, Zion D, Silove D, et al. Placing ethics in the Centre: negotiating new spaces for ethical research in conflict situations. Glob Public Health. 2006;1(3):264-77.

8. Seedat S, Pienaar WP, Williams D, Stein DJ. Ethics of research on survivors of trauma. Curr Psychiatry Rep. 2004:6(4):262-7.

9. Kavukcu N, Altintas KH. The challenges of the health care providers in refugee settings: a systematic review. Prehosp Disaster Med. 2019:1-9.

10. Barrett DH, Ortmann LW, Brown N, DeCausey BR, Saenz C, Dawson A. Public Health Research. In: D HB, L WO, Dawson A, Saenz C, Reis A, Bolan G, editors. Public health ethics: cases spanning the globe. Cham (CH)2016. p. 285-318.

11. Tomczyk B, Lor A. Ethics. In: Townes D, editor. Health in humanitarian emergencies: principles and practice for public health and healthcare practitioners. Cambridge: Cambridge University Press; 2018. p. 68-78.

12. Rousseau C, Kirmayer LJ. From complicity to advocacy: the necessity of refugee research. Am J Bioeth. 2010;10(2):65-7.

13. Ellis BH, Kia-Keating M, Yusuf SA, Lincoln A, Nur A. Ethical research in refugee communities and the use of community participatory methods. Transcult Psychiatry. 2007;44(3):459-81.

14. Dingoyan D, Schulz H, Mosko M. The willingness to participate in health research studies of individuals with Turkish migration backgrounds: barriers and resources. Eur Psychiatry. 2012;27(Suppl 2):S4-9.

15. Emery CR, Lee JY, Kang C. Life after the pan and the fire: depression, order, attachment, and the legacy of abuse among north Korean refugee youth and adolescent children of north Korean refugees. Child Abuse Negl. 2015; 45:90-100.

16. Stevens A, Gilder ME, Moo P, Hashmi A, Toe SET, Doh BB, et al. Folate supplementation to prevent birth abnormalities: evaluating a communitybased participatory action plan for refugees and migrant workers on the Thailand-Myanmar border. Public Health. 2018;161:83-9.

17. Al-Nuaimi S, Aldandashi S, Easa AKS, Saqqur M. Psychiatric morbidity among physically injured Syrian refugees in Turkey. Compr Psychiatry. 2018;80:34-8.

18. Lamkaddem M, Essink-Bot ML, Deville W, Gerritsen A, Stronks K. Health changes of refugees from Afghanistan, Iran and Somalia: the role of residence status and experienced living difficulties in the resettlement process. Eur J Pub Health. 2015;25(6):917-22. 
19. Quach A, Laemmle-Ruff IL, Polizzi T, Paxton GA. Gaps in smiles and services: a cross-sectional study of dental caries in refugee-background children. BMC Oral Health. 2015;15:10.

20. Nyboe L, Bentholm A, Gyllensten AL. Bodily symptoms in patients with post traumatic stress disorder: a comparative study of traumatized refugees, Danish war veterans, and healthy controls. J Bodyw Mov Ther. 2017;21(3): 523-7.

21. Misra SM, Nepal VP, Banerjee D, Giardino AP. Chronic health conditions, physical activity and dietary behaviors of Bhutanese refugees: a Houstonbased needs assessment. J Immigr Minor Health. 2016;18(6):1423-31.

22. Schnyder U, Muller J, Morina N, Schick M, Bryant RA, Nickerson A. A comparison of DSM-5 and DSM-IV diagnostic criteria for posttraumatic stress disorder in traumatized refugees. J Trauma Stress. 2015;28(4):267-74.

23. Nickerson A, Schick M, Schnyder U, Bryant RA, Morina N. Comorbidity of posttraumatic stress disorder and depression in tortured, treatment-seeking refugees. J Trauma Stress. 2017;30(4):409-15

24. Tohme J, Egan JE, Friedman MR, Stall R. Psycho-social correlates of condom use and HIV testing among MSM refugees in Beirut, Lebanon. AIDS Behav. 2016;20(Suppl 3):417-25.

25. Ao T, Shetty S, Sivilli T, Blanton C, Ellis H, Geltman PL, et al. Suicidal ideation and mental health of Bhutanese refugees in the United States. J Immigr Minor Health. 2016;18(4):828-35.

26. Hagaman AK, Sivilli TI, Ao T, Blanton C, Ellis H, Lopes Cardozo B, et al. An investigation into suicides among Bhutanese refugees resettled in the United States between 2008 and 2011. J Immigr Minor Health. 2016;18(4): 819-27

27. Acarturk C, Konuk E, Cetinkaya M, Senay I, Sijbrandij M, Gulen B, et al. The efficacy of eye movement desensitization and reprocessing for posttraumatic stress disorder and depression among Syrian refugees: results of a randomized controlled trial. Psychol Med. 2016;46(12):2583-93.

28. Thela L, Tomita A, Maharaj V, Mhlongo M, Burns JK. Counting the cost of Afrophobia: post-migration adaptation and mental health challenges of African refugees in South Africa. Transcult Psychiatry. 2017;54(5-6):715-32.

29. Ben Farhat J, Blanchet K, Juul Bjertrup P, Veizis A, Perrin C, Coulborn RM, et al. Syrian refugees in Greece: experience with violence, mental health status, and access to information during the journey and while in Greece. BMC Med. 2018;16(1):40.

30. Alemi $\mathrm{Q}$, James S, Montgomery S. Contextualizing afghan refugee views of depression through narratives of trauma, resettlement stress, and coping. Transcult Psychiatry. 2016:53(5):630-53.

31. Kingori C, Esquivel CL, Hassan Q, Elmi A, Mukasa B, Reece M. Recommendations for developing contextually relevant HIV/AIDS prevention strategies targeting African-born immigrants and refugees in the United States. AIDS Patient Care STDs. 2016;30(10):476-83.

32. Tschirhart N, Sein T, Nosten F, Foster AM. Migrant and refugee patient perspectives on travel and tuberculosis along the Thailand-Myanmar border: a qualitative study. PLoS One. 2016;11(8):e0160222.

33. McHenry MS, Umoren R, Dixit A, Holliday R, Litzelman D. Exploring healthcare perspectives of Burmese Chin refugees. J Cult Divers. 2016;23(4): 151-7.

34. Chen YY, Li AT, Fung KP, Wong JP. Improving access to mental health services for racialized immigrants, refugees, and non-status people living with HIV/AIDS. J Health Care Poor Underserved. 2015;26(2):505-18.

35. Tohme J, Egan JE, Stall R, Wagner G, Mokhbat J. HIV prevalence and demographic determinants of unprotected anal sex and HIV testing among male refugees who have sex with men in Beirut, Lebanon. AIDS Behav. 2016;20(Suppl 3):408-16.

36. Jablonka A, Happle C, Grote U, Schleenvoigt BT, Hampel A, Dopfer C, et al. Measles, mumps, rubella, and varicella seroprevalence in refugees in Germany in 2015. Infection. 2016;44(6):781-7.

37. Salt RJ, Costantino ME, Dotson EL, Paper BM. "You are not alone" strategies for addressing mental health and health promotion with a refugee women's sewing group. Issues Ment Health Nurs. 2017;38(4):337-43.

38. Siriwardhana C, Adikari A, Jayaweera K, Sumathipala A. Ethical challenges in mental health research among internally displaced people: ethical theory and research implementation. BMC Med Ethics. 2013;14:13.

39. Jacobsen K, Landau LB. The dual imperative in refugee research: some methodological and ethical considerations in social science research on forced migration. Disasters. 2003;27(3):185-206.

40. Pittaway E, Bartolomei L, Hugman R. 'Stop stealing our stories': the ethics of research with vulnerable groups. J Human Rights Practice. 2010;2(2):229-51.
41. Hyndman J. Managing displacement: refugees and the politics of humanitarianism. Minneapolis: University of Minnesota Press; 2000.

42. Trimble JE, Fisher CB. The handbook of ethical research with ethnocultural populations and communities. London: Sage; 2006.

43. Perry KH. Ethics, vulnerability, and speakers of other languages: how university IRBs (do not) speak to research involving refugee participants. Qual Inq. 2011;17(10):899-912.

44. Odwazny LM, Berkman BE. The "reasonable person" standard for research informed consent. Am J Bioeth. 2017;17(7):49-51.

45. HHS. Revised Common Rule. 2017. https://www.hhs.gov/ohrp/regulationsand-policy/regulations/finalized-revisions-common-rule/index.html. Accessed 16 May 2019.

46. Gabriel PS. Practical and ethical issues in conducting health research with refugees. Vancouver: Simon Fraser University; 2013.

47. Kass NE. An ethics framework for public health. Am J Public Health. 2001; 91(11):1776-82.

48. Bhutta ZA. Beyond informed consent. Bull World Health Organ. 2004;82(10): $771-7$.

49. Racher FE. The evolution of ethics for community practice. J Community Health Nurs. 2007;24(1):65-76.

50. Gbadegesin S, Wendler D. Protecting communities in health research from exploitation. Bioethics. 2006;20(5):248-53.

51. Delemos JL. Community-based participatory research: changing scientific practice from research on communities to research with and for communities. Local Environ. 2006;11(3):329-38.

52. Lee LM. Public health ethics theory: review and path to convergence. J Law Med Ethics. 2012;40(1):85-98.

53. Bledsoe KL, Hopson RK. Conducting ethical research and evaluation in underserved communities. Thousand Oaks: Sage Publications; 2009.

54. National Commission for the Protection of Human Subjects of Biomedical and Behavioral Research. The Belmont report: Ethical principles and guidelines for the protection of human subjects of research. 1979. Retrieved from https://www.hhs.gov/ohrp/regulations-and-policy/belmont-report/ read-the-belmont-report/index.html.

\section{Publisher's Note}

Springer Nature remains neutral with regard to jurisdictional claims in published maps and institutional affiliations.
Ready to submit your research? Choose BMC and benefit from:

- fast, convenient online submission

- thorough peer review by experienced researchers in your field

- rapid publication on acceptance

- support for research data, including large and complex data types

- gold Open Access which fosters wider collaboration and increased citations

- maximum visibility for your research: over $100 \mathrm{M}$ website views per year

At $\mathrm{BMC}$, research is always in progress.

Learn more biomedcentral.com/submissions 